\title{
USO DE ENSAIOS DE MICROESCLEROMETRIA INSTRUMENTADA NO ESTUDO DAS PROPRIEDADES DA AUSTENITA EXPANDIDA
}

\author{
Fernando Luis Sato ' \\ Luis Armando Espitia ' \\ Carlos Eduardo Pinedo ${ }^{2}$ \\ André Paulo Tschiptschin '
}

\section{Resumo}

A resistência à corrosão do aço inoxidável austenítico AISI 316 é usualmente acompanhada por propriedades mecânicas limitadas dessa liga. A austenita expandida, ou Fase-S, supersaturada em nitrogênio, endurece superficialmente o material, sem a formação de precipitados e consequente prejuízo da passivação, efeitos comumente observados nos métodos convencionais de endurecimento superficial. Caracterizações mecânicas dessa camada por ensaios de microesclerometria instrumentada fornecem um conjunto de dados empíricos relevantes para a compreensão e modelagem de fenômenos tribológicos atuantes em um dado sistema mecânico. $O$ presente trabalho apresenta os resultados de uma série de ensaios de microesclerometria instrumentada realizados em amostras de aço AISI 316 nitretadas a plasma por $20 \mathrm{~h}$ em forno de corrente contínua, utilizando a tecnologia de tela ativa, temperatura de $400^{\circ} \mathrm{C}$ e atmosfera formada por três partes de nitrogênio para uma parte de hidrogênio $\left(3 \mathrm{~N}_{2}: \mathrm{IH}_{2}\right)$. Também são discutidos a caracterização das amostras e demais resultados, como o reduzido coeficiente de atrito $(<0, \mathrm{I})$ e a ausência de falha adesiva da camada.

Palavras-chave: Microesclerometria; Austenita expandida; Fase-S.

\section{INSTRUMENTED MICROSCRATCH TESTS USAGE FOR STUDY OF EXPANDED AUSTENITE PROPERTIES}

\begin{abstract}
Corrosion resistance and poor mechanical properties are both characteristics of AISI 316 austenitic stainless steel. Nitrogen supersaturated expanded austenite, or S-phase, promotes surface hardening of the alloy without the formation of undesirable that can reduce passivation properties. Mechanical characterization of this layer using instrumented microscratch tests gives an important set of empirical data useful for comprehension and modeling of tribological phenomena occurring in mechanical system. This work presents results from a series of instrumented microscratch tests performed on Low Temperature Plasma Nitrided (LTPN) AISI 316 stainless steel samples with an expanded austenite layer. The specimens were produced by $20 \mathrm{~h}$ active screen plasma nitriding treatment, done in direct current reactor at $400^{\circ} \mathrm{C}$ in an atmosphere containing three parts of nitrogen for one part of hydrogen $\left(3 \mathrm{~N}_{2}: I \mathrm{H}_{2}\right)$. The reduced friction coefficient $(<0, \mathrm{I})$ between the indenter and the expanded austenite layer observed in the initial stage of scratch test and the absence of adhesive failure along the test are also discussed.
\end{abstract}

Keywords: Microscratch test; Expanded austenite; S-phase.

\section{INTRODUÇÃo}

A demanda por aços inoxidáveis vem aumentando nas últimas décadas em razão da necessidade de se construir máquinas e equipamentos com materiais que possuam elevada resistência à corrosão [I]. Indústrias como a química, de energia, alimentos, bebidas, farmacêuticas e, mais recentemente, até da construção civil [2] têm visto na inoxidabilidade dessa classe de aços uma oportunidade de reduzir significativamente os custos de manutenção e aumentar o ciclo de vida de equipamentos e estruturas, alinhando-se a uma visão moderna de sustentabilidade.

Entretanto, as propriedades mecânicas da austenita nesses aços, em especial do AISI 316 [3], restringem as

'Departamento de Engenharia Metalúrgica e de Materiais, Escola Politécnica da Universidade de São Paulo - Poli-USP, São Paulo, SP, Brasil.

E-mail: flsato@usp.br

${ }^{2}$ Heat Tech Tecnologia em Tratamento Térmico e Engenharia de Superfície, Mogi das Cruzes, SP, Brasil. 
aplicações dessas ligas em sistemas tribologicamente agressivos devido à necessidade de maior dureza superficial para melhor resistência ao desgaste [4].

$\mathrm{O}$ estudo das relações entre a microestrutura e as propriedades dos materiais talvez seja a chave para possibilitar a combinação de características mecânicas, muitas vezes conflitantes, tais como elevadas dureza, tenacidade e resistência à corrosão combinadas a um coeficiente de atrito bastante baixo, combinação de propriedades não muito simples de se obter pelos métodos convencionais de endurecimento. Nos tratamentos de nitretação mais usuais $\left(>500^{\circ} \mathrm{C}\right)$ de aços inoxidáveis, a elevação de dureza superficial é acompanhada por uma diminuição na resistência à corrosão da liga devido à intensa precipitação de nitretos do tipo $(\mathrm{Cr}, \mathrm{Fe}) \mathrm{Ne}$ e $(\mathrm{Cr}, \mathrm{Fe})_{2} \mathrm{~N}$, além de carbonetos do tipo $(\mathrm{Cr}, \mathrm{Fe})_{23} \mathrm{C}_{6}[5]$.

As técnicas baseadas no uso do plasma são bons exemplos de tratamentos para obtenção de características superficiais diferenciadas, pois possibilitam o controle sobre a metalurgia da camada tratada [6], permitindo a combinação de propriedades virtualmente díspares no sistema camadasubstrato, de forma planejada e economicamente viável, que visa atender as demandas para determinadas aplicações mecânicas.

Em meados da década de 1980 [7], ao submeter aços inoxidáveis a tratamentos termoquímicos assistidos por plasma, notou-se a ocorrência de uma expansão da estrutura cristalina que lhe conferia propriedades específicas tais como elevada dureza, isenção de precipitados e manutenção da resistência à corrosão da liga original. A nova fase, produzida pela adição de intersticiais como carbono e/ou nitrogênio em níveis muito acima do limite de solubilidade na austenita, foi descrita como uma solução sólida supersaturada, posteriormente denominada austenita expandida, ou Fase-S. Desde então, essa estrutura metaestável, isenta de precipitados (observáveis por técnicas convencionais de microscopia), vem sendo investigada, no que se refere à sua natureza física e às suas propriedades.

Não foram encontrados trabalhos na literatura sobre o comportamento tribológico de aços inoxidáveis austeníticos nitretados a plasma em baixa temperatura, em ensaios de microesclerometria linear instrumentada. Este trabalho se propõe a estudar as propriedades tribológicas da austenita expandida por meio de resultados obtidos experimentalmente em ensaios de microesclerometria instrumentada, analisando as características do risco produzido na superfície das amostras, utilizando técnicas analíticas convencionais, tais como medidas de dureza e análise metalográfica. Pela observação das interações do penetrador durante o riscamento, pode-se determinar a carga crítica para o início de falha coesiva da camada, bem como avaliar os demais resultados extraídos ao longo dos ensaios, como as forças tangencial e normal, nível de emissões acústicas no rompimento frágil da camada, coeficiente de atrito e profundidade atingida pelo penetrador. Esses dados empíricos se mostram relevantes para a compreensão e modelagem de fenômenos tribológicos atuantes em um dado sistema mecânico, colocando a microesclerometria instrumentada como uma interessante ferramenta para os estudos direcionados à Engenharia de Superfície.

\section{METODOLOGIA}

\section{I Amostras e Equipamentos}

As amostras foram confeccionadas a partir de uma barra de aço AISI 316 comercial, laminada a quente, cuja composição típica é apresentada na Tabela I.

As amostras foram usinadas e retificadas na forma de discos com $25 \mathrm{~mm}$ de diâmetro por $5 \mathrm{~mm}$ de espessura, conforme mostrado na Figura Ib. Após acabamento dado por lixas de carbeto de silício com granulometria até 600 e polimento com pasta de diamante até $3 \mu \mathrm{m}$, as peças foram submetidas aos tratamentos superficiais de nitretação por plasma. Adotando-se uma temperatura de tratamento de $400^{\circ} \mathrm{C}$ e uma composição atmosférica formada por três partes de nitrogênio para cada parte de hidrogênio $\left(3 \mathrm{~N}_{2}: \mathrm{IH}_{2}\right)$, os tratamentos foram realizados por $20 \mathrm{~h}$.

O equipamento utilizado neste trabalho foi o UMT Multi-Specimen Test System (mostrado na Figura la), um tribômetro universal que permite diferentes configurações para teste de uma variedade de amostras e pode ser adaptado para execução de diversos tipos de ensaios [8] com interesse tribológico, tais como pino-disco, esfera-disco, esfera-esfera, disco-disco, etc. Como características principais, pode-se citar o percurso de movimento vertical de $150 \mathrm{~mm}$, precisão instrumental de $50 \mathrm{~nm}$ para medições de atrito, células

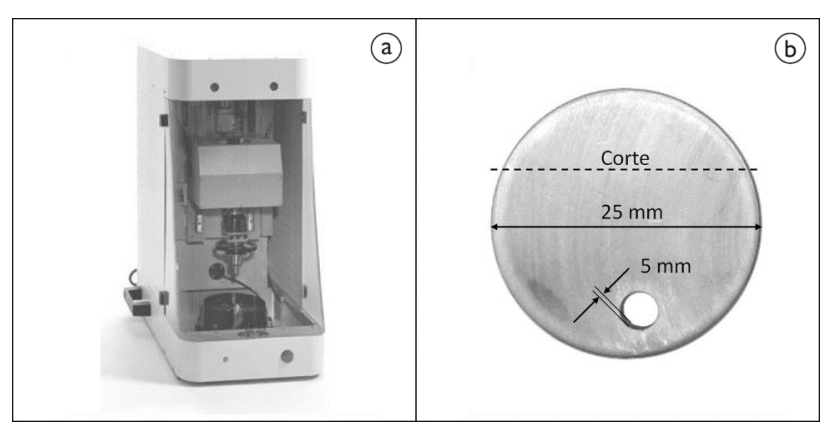

Figura I. (a) Equipamento para realização dos ensaios de microesclerometria instrumentada; e (b) amostra nitretada com dimensões e esquema de corte.

Tabela I. Composição química típica dos aços AISI 3 I6 [3]

\begin{tabular}{ccccccccc}
\hline Elemento & $\mathbf{C}$ & $\mathbf{M n}$ & $\mathbf{S i}$ & $\mathbf{S}$ & $\mathbf{P}$ & $\mathbf{C r}$ & $\mathbf{N i}$ & $\mathbf{M o}$ \\
\hline$\%$ em peso & $<0,03$ & 2 & 0,5 & $<0,01$ & $<0,02$ & 17 & 12 & 2,5 \\
\hline
\end{tabular}


de cargas independentes capazes de registrar as cargas aplicadas e forças desenvolvidas durante o ensaio, com precisão de miligramas e sensor ativo de força normal que mantém constante a intensidade da força aplicada ao longo do teste. Os dados podem ser exibidos em tempo real ou armazenados para futuro tratamento no microcomputador que controla o equipamento. A unidade de teste possui uma estrutura de ferro fundido com alta capacidade de absorção de vibrações e sistema motorizado de posicionamento lateral e vertical do indentador.

O equipamento foi preparado para utilização de um penetrador cônico de diamante, tipo Rockwell C, com ângulo de vértice de $120^{\circ}$ e raio de $200 \mu \mathrm{m}$.

Para análise da seção transversal, a amostra foi cortada com disco diamantado de forma a permitir a observação da espessura e regularidade da camada, preservando-se o resto da amostra para a eventualidade de confirmação dos resultados dos ensaios mecânicos.

A nitretação a plasma, utilizando a técnica de tela ativa ("Active Screen Plasma"), foi feita em um forno de corrente contínua Klöckner lonon 60 kVA.

Para confirmação da presença de austenita expandida, foi realizado ensaio de difração de raios- $X$ em um equipamento $X$ 'Pert pelo método de varredura simples, contínua, usando tubo de cobre $(\lambda=0,15419 \mathrm{~nm})$, com 40-40 kV/mA, a partir de um ângulo inicial de $30^{\circ}$ até $110^{\circ}$, com passo de $0,02^{\circ}$ e tempo total de análise de $50 \mathrm{~s}$.

As medidas de dureza foram realizadas em um durômetro Shimadzu, série HMV-2, com carga de 10 g e as análises metalográficas, em microscópio ótico Olympus, modelo BX60M, e microscópio eletrônico de varredura Philips, modelo XL-30.

\subsection{Ensaios e Análises}

Inicialmente, as amostras foram submetidas à análise de difração de raios- $X$ apenas para a confirmação da presença de austenita expandida, sem a aplicação de modelos de refinamento. Os resultados serviram para o cálculo estimado do teor de nitrogênio dissolvido na fase segundo a relação proposta por Picard [9] que correlaciona a diferença entre os parâmetros de rede da austenita $\left(a_{\gamma}\right)$ e da austenita expandida $\left(a_{\gamma N}\right)$ com o teor de nitrogênio $\left(C_{N}\right)$ em percentual atômico no ferro. $O$ coeficiente de expansão da estrutura, ou constante de Vegard, é dado por $A=0,00078 \mathrm{~nm} / \mathrm{at} . \% \mathrm{~N}$ na austenita virgem, conforme Equação $I$.

$$
a_{\gamma N}=a_{\gamma}+A C_{N}
$$

A Equação I, demonstra a relação entre o parâmetro de rede da austenita expandida e $\circ$ teor de nitrogênio na fase.

A superfície de austenita expandida foi analisada por microscopia eletrônica de varredura e submetidas aos testes de microdureza Vickers com carga de $10 \mathrm{~g}$. Os parâmetros do microesclerômetro foram ajustados para realizar uma impressão de $5 \mathrm{~N}$ no início do teste, seguindo com o riscamento por $10 \mathrm{~mm}$ durante $60 \mathrm{~s}$, com taxa de carregamento crescente de $0,5 \mathrm{~N}$ a 10,5 N ao longo do teste. Os parâmetros estabeleceram $0,167 \mathrm{~mm} / \mathrm{s}$ para a velocidade de deslocamento linear do penetrador e $0,167 \mathrm{~N} / \mathrm{s}$ para a taxa de carregamento, conforme Norma ASTM CI62405 [10].

Previamente ao teste, as amostras foram imersas em acetona e deixadas no aparelho de ultrassom por 5 minutos para serem removidos quaisquer resíduos de lubrificante ou partículas exógenas aderidas à superfície. $O$ penetrador do microesclerômetro, após ter sido inspecionado no MEV para confirmação de sua integridade, foi limpo com um tecido embebido em acetona.

$\mathrm{Na}$ superfície de cada uma das amostras foram realizados cinco riscos paralelos, iniciando da extremidade para o centro da seção circular. Foi preservada uma distância de pelo menos $1 \mathrm{~mm}$ entre os riscos. A Figura 2 ilustra as configurações para a realização do teste, disposição e direção dos riscos.

Cada um dos riscos foi avaliado por microscopia ótica, com aumento de $500 \mathrm{X}$, para determinação das regiões onde foi possível detectar as primeiras trincas na camada. A distância entre a origem do risco e o início de trincamento foi medida com o micrômetro do próprio microscópio, com precisão de $0,05 \mathrm{~mm}$. Para determinação da força crítica de trincamento, essas distâncias foram correlacionadas aos
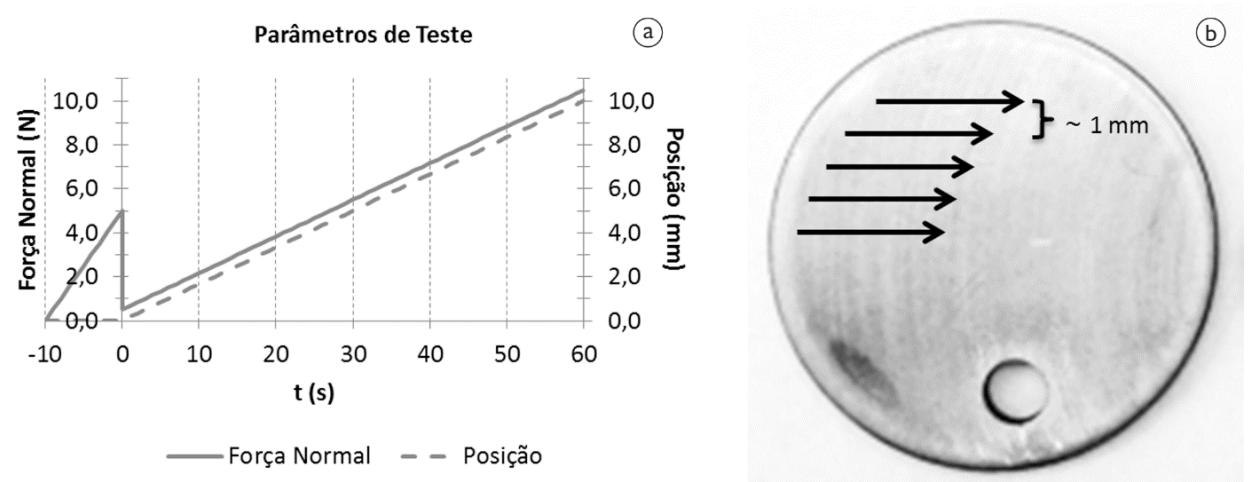

Figura 2. Esquema para teste de microesclerometria, mostrando (a) o posicionamento e a força aplicada no penetrador ao longo do tempo; e (b) disposição e direção dos riscos. 
respectivos registros da força normal aplicada na posição específica.

As amostras foram, então, cortadas transversalmente por um disco diamantado para avaliação metalográfica e determinação da espessura de camada na face riscada. Os segmentos foram embutidos em baquelite, lixados em carbeto de silício até granulometria de 1.200 , polidas com pasta de diamante até granulometria de $\mathrm{I} \mu \mathrm{m}$ e atacadas com reagente de Vilela por I h.

\section{RESULTADOS}

As amostras numeradas como I e 3 foram submetidas à análise por difração de raios-X para confirmação da presença de austenita expandida. Os dados foram utilizados para $\circ$ cálculo da estimativa do teor de nitrogênio em solução sólida, conforme mostra os difratogramas da Figura 3. Os valores utilizados no cálculo do parâmetro de rede são apresentados na Tabela 2.

Usando a relação proposta por Picard, foi possível calcular o valor teórico da concentração de nitrogênio dissolvido na camada de austenita expandida, obtendo-se uma concentração estimada de nitrogênio na austenita expandida de $C_{N}=34,8$ at. $\% \mathrm{~N}$ para a amostra I e $C_{N}=34,2$ at. $\% \mathrm{~N}$

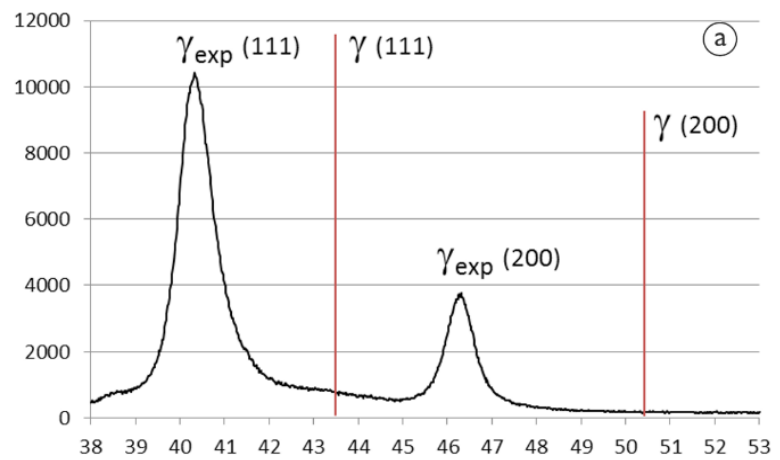

para a amostra 3. Neste cálculo, não foram considerados os efeitos da tensão atuante na camada.

Os valores de microdureza Vickers medidos no topo da superfície nitretada, com carga de $10 \mathrm{~g}$, são mostrados na Tabela 3. Vale notar que a profundidade atingida pelo penetrador durante essas medidas foi calculada, resultando em 0,9 $\mu \mathrm{m}$ na amostra I e $0,7 \mu \mathrm{m}$ na amostra 3 . Tais valores são ligeiramente superiores a $10 \%$ da profundidade da camada de austenita expandida, com aproximadamente $6 \mu \mathrm{m}$, indicando que os resultados podem ter sido influenciados pela dureza do substrato.

Previamente aos ensaios no microesclerômetro, foi avaliada a superfície das amostras no MEV, observando-se (Figura 4) o relevo causado pela movimentação relativa dos grãos austeníticos em decorrência das tensões desenvolvidas pela supersaturação do reticulado cristalino da austenita pelo nitrogênio.

Durante o ensaio de microesclerometria instrumentada, foram registradas as forças tangencial e normal, posição do penetrador e a profundidade de risco. $O$ coeficiente de atrito aparente é calculado pela relação entre as forças tangencial e normal, instantaneamente registradas ao longo do ensaio.

A Figura 5 mostra os resultados de dois dos riscos obtidos no ensaio de microesclerometria instrumentada para as amostras I e 3. Observa-se no gráfico da

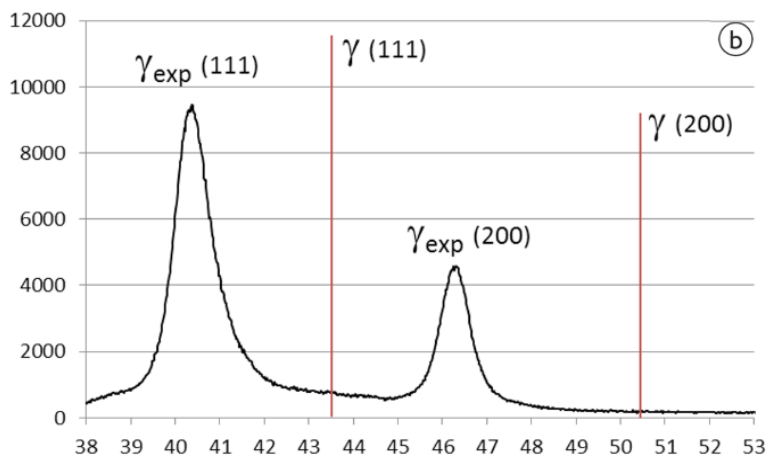

Figura 3. Difratogramas das amostras (a) I e (b) 3.

Tabela 2. Cálculo dos parâmetros de rede da austenita expandida das amostras I e 3

\begin{tabular}{|c|c|c|c|c|c|c|c|c|c|c|c|}
\hline Amostra & Fase & $\mathbf{h}$ & $\mathbf{k}$ & I & $2 \theta$ & $\theta$ (rad) & $\operatorname{sen} \theta$ & $\lambda(\mathbf{n m})$ & $\mathbf{n}$ & $\mathrm{d}(\mathrm{nm})$ & $a(n m)$ \\
\hline \multirow[t]{4}{*}{ I } & $\gamma_{\exp }$ & 1 & 1 & I & 40,32 & 20,16 & 0,3446 & 0,1542 & I & 0,2237 & 0,3875 \\
\hline & $\gamma$ & 1 & 1 & I & 43,51 & 21,76 & 0,3706 & 0,1542 & I & 0,2080 & 0,3603 \\
\hline & $\gamma_{\exp }$ & 2 & 0 & 0 & 46,31 & 23,16 & 0,3932 & 0,1542 & I & 0,1961 & 0,3921 \\
\hline & $\gamma$ & 2 & 0 & 0 & 50,38 & 25,19 & 0,4256 & 0,1542 & I & $0,|8| \mid$ & 0,3623 \\
\hline \multirow[t]{4}{*}{3} & $\gamma_{\exp }$ & 1 & 1 & I & 40,37 & 20,19 & 0,3451 & 0,1542 & I & 0,2234 & 0,3870 \\
\hline & $\gamma$ & I & I & I & 43,51 & 21,76 & 0,3706 & 0,1542 & I & 0,2080 & 0,3603 \\
\hline & $\gamma_{\exp }$ & 2 & 0 & 0 & 46,29 & 23,15 & 0,3931 & 0,1542 & I & $0,|96|$ & 0,3923 \\
\hline & $\gamma$ & 2 & 0 & 0 & 50,38 & 25,19 & 0,4256 & 0,1542 & I & $0,|8| \mid$ & 0,3623 \\
\hline
\end{tabular}

Tabela 3. Valores de dureza Vickers medidos diretamente sobre a camada de austenita expandida com carga de $10 \mathrm{~g}$

\begin{tabular}{cccccccccccc}
\hline Medida & I & $\mathbf{2}$ & $\mathbf{3}$ & $\mathbf{4}$ & $\mathbf{5}$ & $\mathbf{6}$ & $\mathbf{7}$ & $\mathbf{8}$ & $\mathbf{9}$ & $\mathbf{1 0}$ & Média \\
\hline Am. I & 1126 & 872 & 838 & 946 & 1296 & 1126 & 1030 & 1030 & 1076 & 1126 & 1038 \\
Am. 3 & 908 & 1296 & 1362 & 1362 & 1235 & 1362 & 1126 & 1779 & 1433 & 1509 & 1337 \\
\hline
\end{tabular}



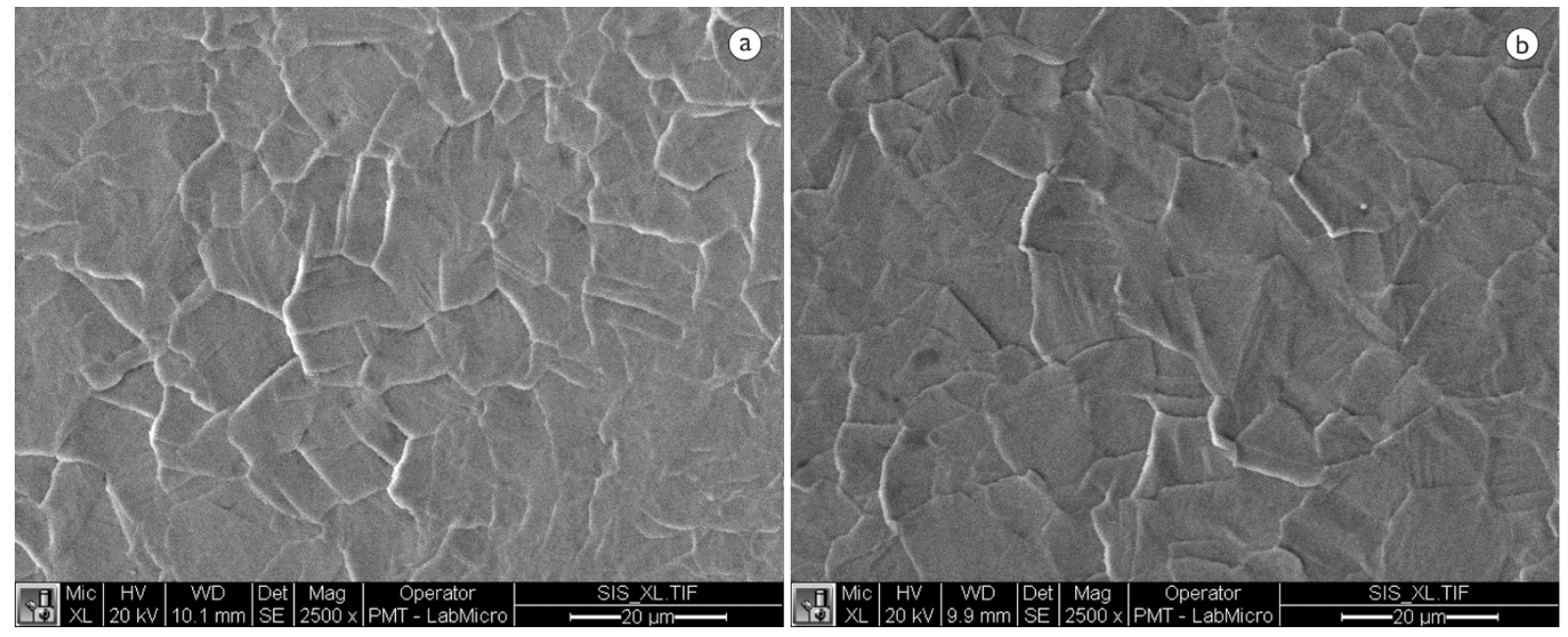

Figura 4. Imagem da superfície das amostras (a) I e (b) 3 no MEV (sem ataque). O relevo observado decorre da movimentação relativa de grãos na superfície, ocasionada pelas tensões residuais compressivas desenvolvidas durante nitretação.
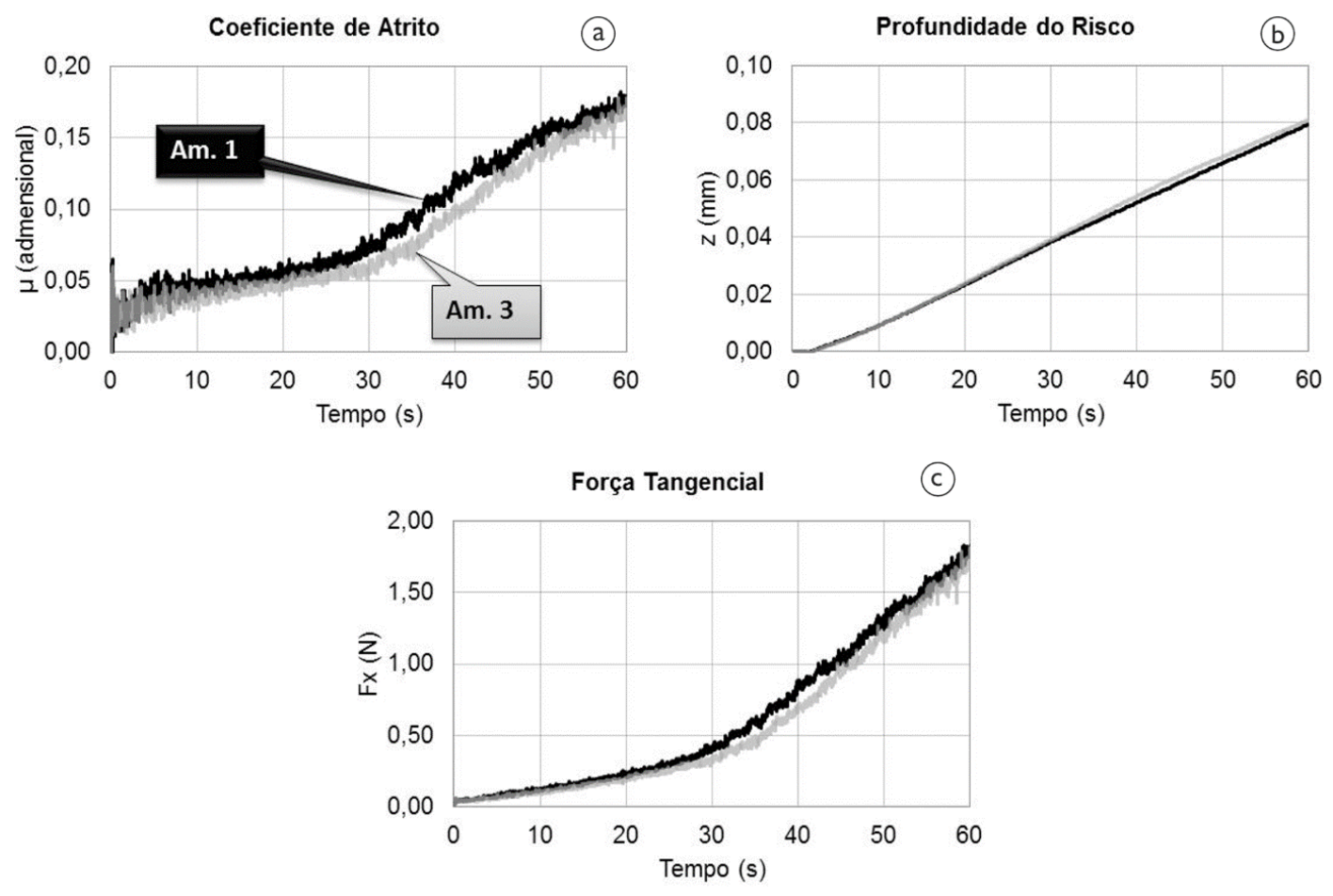

Figura 5. Resultados dos ensaios de microesclerometria instrumentada obtidos em riscos das amostras I e 3.

Figura 5a que o coeficiente de atrito se mantém em níveis muito baixos até cerca da metade da duração do teste, enquanto que a força tangencial $F_{x}$ varia linearmente nesse intervalo até atingir cerca de $0,5 \mathrm{~N}$, conforme mostra a Figura $5 \mathrm{c}$.

Todos os riscos foram documentados por meio de microscopia ótica em três regiões: no início, onde as primeiras trincas na camada começam a aparecer e no final do risco. A Figura 6 apresenta as imagens dos riscos produzidos nas amostras I e 3 referentes aos resultados mostrados na Figura 5.
As forças normais, relativas a cada uma das posições indicadas como críticas, foram relacionadas na Tabela 4, bem como as forças normais críticas do sistema estudado, calculadas pela média aritmética das forças normais correspondentes às regiões onde as trincas na camada se iniciavam.

Posteriormente aos ensaios de riscamento, as amostras foram cortadas e analisadas metalograficamente quanto à homogeneidade de recobrimento e para determinação de sua espessura média de camada. Ambas apresentaram camadas homogêneas ao longo de toda superfície com espessura média em torno dos $6 \mu \mathrm{m}$ (Figura 7). 

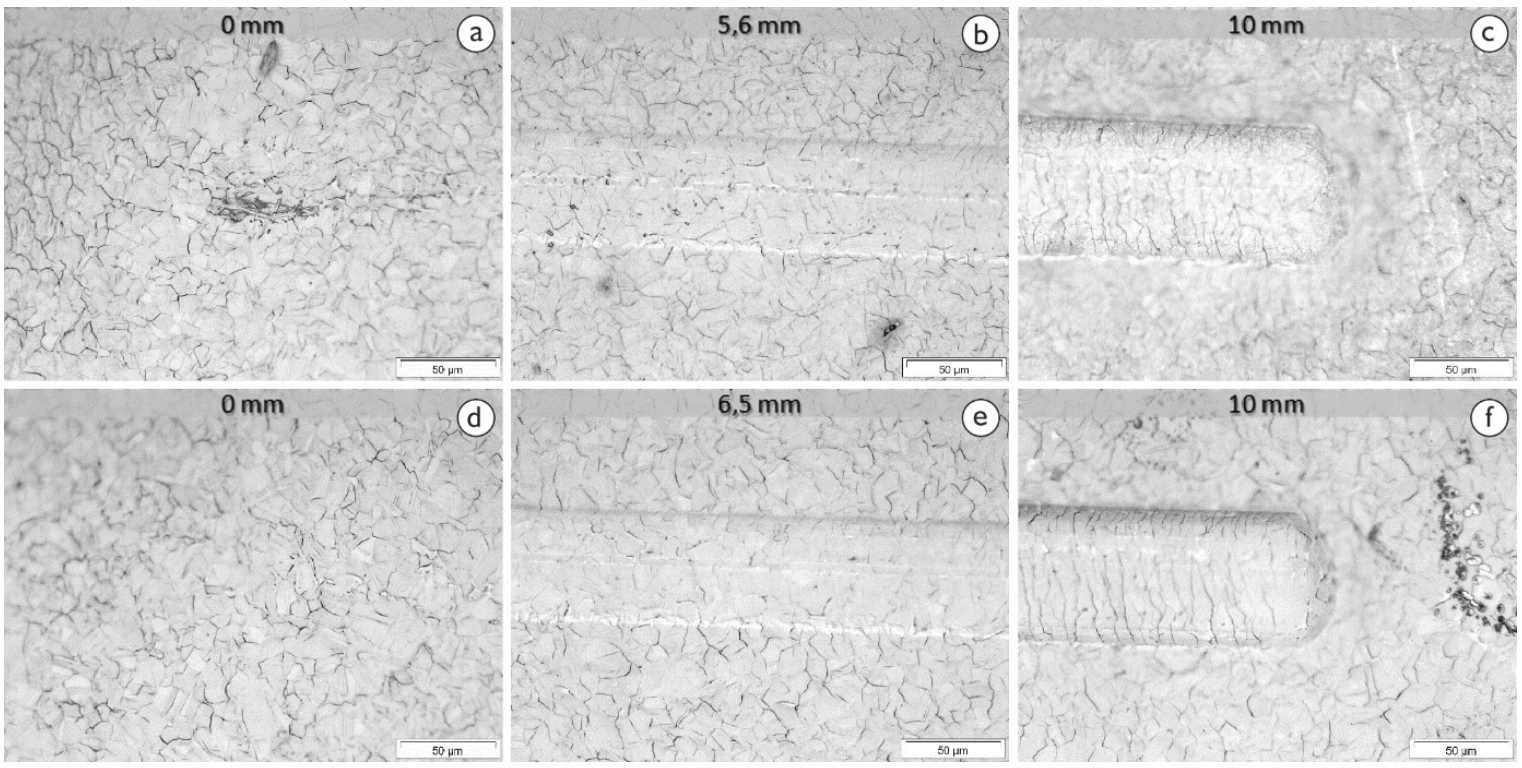

Figura 6. Micrografia do início (a;d), zona crítica (b;e) e final (c;f) dos riscos produzidos sobre a camada de austenita expandida das amostras I e 3 , respectivamente.
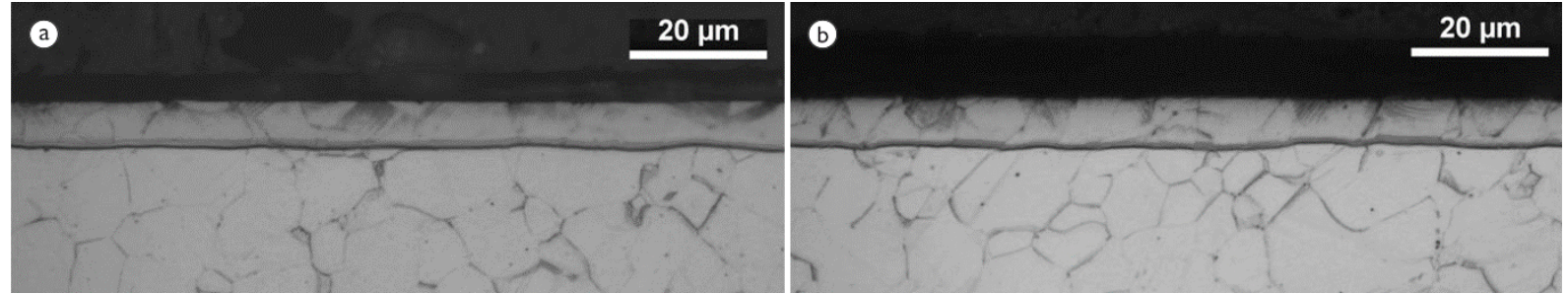

Figura 7. Seção transversal da (a) amostra I e (b) 3, evidenciando a homogeneidade de recobrimento, com espessuras de camada em torno dos $6 \mu \mathrm{m}$. Ataque: Vilella.

Tabela 4. Forças normais críticas determinadas na análise dos riscos

\begin{tabular}{ccccccc}
\hline Risco & I & $\mathbf{2}$ & $\mathbf{3}$ & $\mathbf{4}$ & $\mathbf{5}$ & Média \\
\hline Amostra I $(\mathrm{N})$ & 5,7 & 5,8 & 6,0 & 6,3 & 5,9 & $\mathbf{5 , 9}$ \\
Amostra 2 $(\mathrm{N})$ & 6,6 & 6,6 & 6,7 & 6,8 & 6,6 & $\mathbf{6 , 7}$ \\
\hline
\end{tabular}

\section{DISCUSSÃO}

Não foram encontrados na literatura técnica outros estudos sobre a utilização de ensaios de microesclerometria instrumentada na investigação das propriedades tribológicas da austenita expandida. A Norma ASTM CI624-05 [10], que trata desse ensaio em materiais metálicos com recobrimentos do tipo cerâmico, foi empregada com sucesso na padronização dos ensaios e análise dos resultados, bem como na investigação das propriedades da camada de austenita expandida.

$\mathrm{Na}$ formação da camada de austenita expandida não há a presença de uma interface abrupta, ou mecânica, entre os grãos do substrato e da camada superficial. Assim, o desplacamento, que para os filmes cerâmicos serve como referência na determinação experimental da força normal crítica, não ocorre da mesma maneira, pois o tratamento termoquímico, formador da camada de austenita expandida, induz um gradiente de composição responsável pela adesão ideal ao substrato, praticamente impossibilitando o destacamento da camada.

Considerando os resultados nos ensaios, pode-se observar que o indentador desliza com coeficiente de atrito constante e muito baixo sobre a camada de austenita expandida, mesmo com a carga crescente. O rompimento da camada se inicia quando a força normal atinge valores críticos, próximos aos $6 \mathrm{~N}$ na amostra I e 6,5 $\mathrm{N}$ na amostra 3 , quando surgem as primeiras trincas observáveis por microscopia ótica (Figura 6) na trilha do risco. O evento pode ser detectado por técnicas auxiliares como a emissão acústica dos rompimentos frágeis na camada, ou até mesmo pela inflexão da curva do coeficiente de atrito obtida.

A Figura 8 mostra um comparativo entre resultados obtidos para a austenita com e sem tratamento, realizado a uma taxa de carregamento de $0,353 \mathrm{~N} / \mathrm{s}$ e velocidade de riscamento de $0,159 \mathrm{~mm} / \mathrm{s}$. Verifica-se que a inflexão da curva 
Coeficiente de Atrito

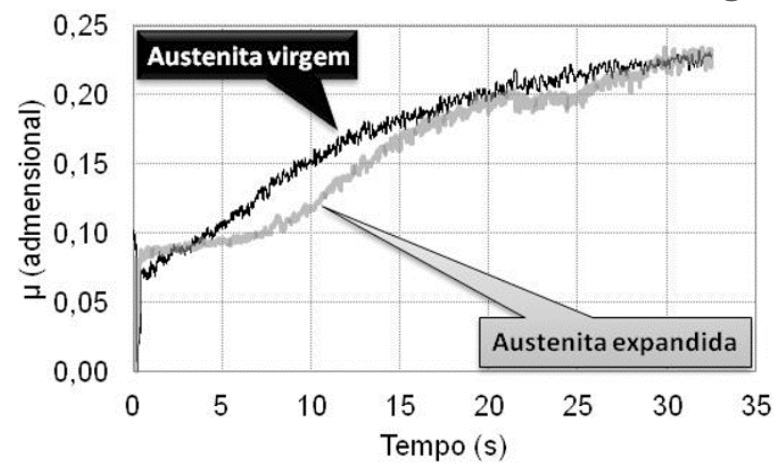

Emissão Acústica

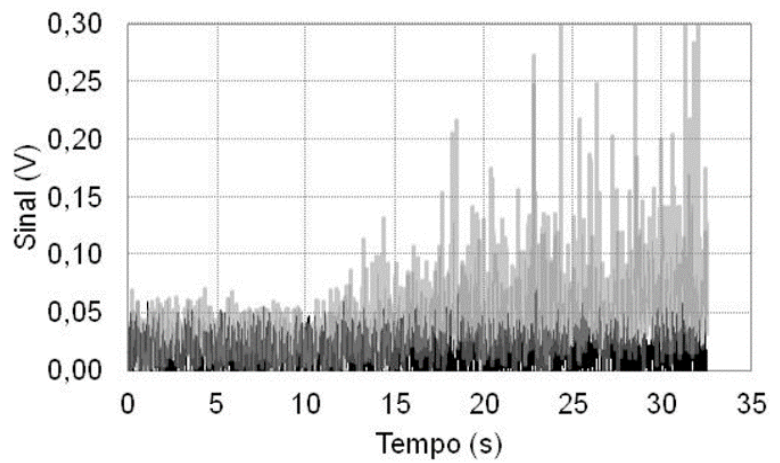

Figura 8. Comparação entre (a) os coeficientes de atrito aparente obtidos em ensaio de microesclerometria instrumentada para a austenita expandida e sem tratamento nos aços AISI 316; (b) e comparação entre sinais de emissão acústica detectada para os mesmos materiais.

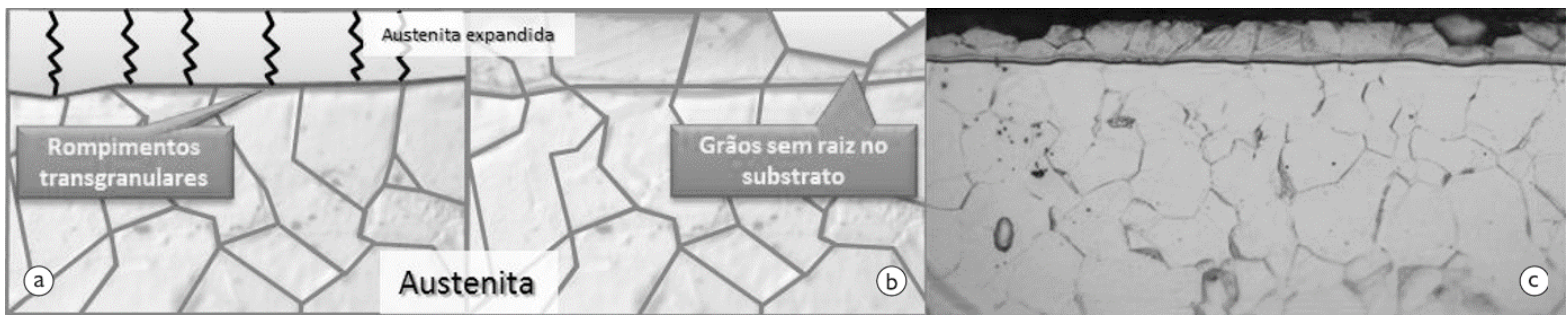

Figura 9. (a) Esquema de trincamento transgranular; e (b) intergranular, promovendo o destacamento na camada de austenita expandida. Micrografia da seção transversal da amostra I. Ataque: Vilella.

do coeficiente de atrito obtido para a austenita expandida (Figura 8a) coincide com o aumento de intensidade de emissão acústica (Figura 8b), indicando falha coesiva da camada.

Holmberg e Matthews citam o coeficiente de atrito entre o diamante e o metal como sendo de até $0, \mathrm{I}[\mathrm{II}] \mathrm{e}$, de fato, no início dos ensaios o valor permanece bastante abaixo desse limite, em torno de 0,05 , até o aparecimento das primeiras trincas na trilha desgastada, em torno dos $10 \mathrm{~s}$ de ensaio, concomitantemente ao aumento dos sinais de emissão acústica que também podem ser registrados como forma auxiliar de detecção dessa transição. $O$ intervalo em que o coeficiente permanece constante é desejável para aplicações que demandem baixo atrito e pode servir de indicativo para a integridade da camada superficial. Contrariamente, o risco sobre a austenita virgem mostra um coeficiente de atrito aparente crescente do início ao fim do ensaio.

Analisando os destacamentos encontrados ao longo do risco, verifica-se que não houve falha adesiva da camada ao substrato, mas a propagação intergranular de trincas sob os grãos que não possuem raiz no substrato austenítico original. $O$ esquema mostrado na Figura 9a ilustra a presença de trincas transgranulares que atravessam a camada na trilha desgastada. A Figura $9 \mathrm{~b}$ ilustra a ocorrência de trincas intergranulares que acabam por promover o destacamento de pequenos grãos não enraizados na matriz austenítica, tal como mostrado na micrografia da Figura 9 c, cuja seção transversal evidencia regiões de destacamento na camada.
No final do teste, mesmo com o rompimento frágil de toda a espessura da camada, momento em que o penetrador entra em contato direto com o substrato austenítico menos resistente, o destacamento não ocorre devido à excelente adesão ao substrato. É possível observar o trincamento gradativo em três estágios distintos, sem nenhuma evidência de desplacamento da camada superficial.

Embora a dureza da austenita expandida ao nitrogênio seja bastante elevada, os resultados mostram que a fase possui uma boa tenacidade, haja vista que não terem sido observadas trincas frágeis no início do riscamento, mesmo quando a superfície aparece visivelmente deformada. Essa tenacidade se contrapõe à dos recobrimentos cerâmicos, notadamente menor, além da maior aderência da camada superficial ao substrato, favorecida pelo gradiente de concentração gerado na formação da austenita expandida, indicando uma possível melhora no desempenho sob impacto e, consequentemente, ampliação da vida útil na aplicação.

\section{CONCLUSÃO}

Foram realizados, de forma inédita, ensaios de microesclerometria instrumentada para o estudo tribológico da austenita expandida, com base em Norma já existente, ASTM Cl624-05 [10], para avaliação de recobrimentos do tipo cerâmico ( $\mathrm{TiNe} \mathrm{CrN}$ ). Verificou-se que, diferentemente do comportamento dos recobrimentos cerâmicos, não houve desplacamento da camada de austenita expandida 
no riscamento, devido, entre outras razões, à indução de gradientes de concentração de nitrogênio em solução sólida intersticial, promovida pela nitretação a plasma com tela ativa. Dessa forma, a camada de austenita expandida pode ser considerada uma continuidade dos grãos originais do substrato, garantindo uma adesão ideal, que pode ser de grande valor para as aplicações cuja solicitação não ultrapasse o limite de resistência da camada formada.

A despeito de sua elevada dureza, a austenita expandida apresenta boa tenacidade, não evidenciando rompimentos frágeis antes de razoável deformação plástica induzida pelo penetrador, atuando sob forças normais críticas ao redor de 6,0 N. Diferentemente da tendência sempre crescente verificada no material sem tratamento, o coeficiente de atrito, entre a austenita expandida e o penetrador de diamante, tende a se estabilizar em torno de 0,05 , valor surpreendentemente baixo, o que torna promissora, para várias aplicações tribológicas, a alternativa de endurecimento superficial dos aços AISI 316, pela formação de austenita expandida por nitretação a plasma com tela ativa.

\section{REFERÊNCIAS}

I Iron \& Steel Society. Steel products manual: stainless steels. Warrendale; 1999.

2 Rossi B. Discussion on the use of stainless steel in constructions in view of sustainability. Thin-walled Structures. 2014;83:182-189. http://dx.doi.org//0.1016/j.tws.2014.01.02I.

3 Farrar JCM. The alloy tree: a guide to low-alloy steels, stainless steels and nickel-base alloys. Cambridge: Woodhead; 2004.

4 Leyland A, Matthews A. Design criteria for wear-resistant nanostructured and glassy-metal coatings. Surface and Coatings Technology. 2004; 177-178:317-324. http://dx.doi.org/10.1016/j.surfcoat.2003.09.01।.

5 Pinedo CE. Tecnologia de plasma na geração de camadas duplex em aços inox austeníticos. Metalurgia \& Materiais. 2009;65:407-409.

6 Pinedo CE. Nitretação por plasma de aços inoxidáveis. Metalurgia \& Materiais. 2004;60:162-164.

7 Dong H. S-phase surface engineering of $\mathrm{Fe}-\mathrm{Cr}$, $\mathrm{Co}-\mathrm{Cr}$ and Ni-Cr alloys. International Materials Reviews. 2010;55(2):65-98. http://dx.doi.org//0.1 179/095066009XI2572530170589.

8 Center for Tribology Research - CETR. UMT multi-specimen test system: hardware installation \& applications manual. Campbell; 2009.

9 Picard S, Memet JB, Sabot R, Grosseau-Poussard JL, Rivière JP, Meilland R. Corrosion behaviour, microhardness and surface characterization of low energy, high current ion implanted austenitic stainless steel. Materials Science and Engineering A. 200 I;303(I-2): I63-I72. http://dx.doi.org/I0. I0I6/S092I-5093(00)0 I84I-4.

10 American Society for Testing and Materials - ASTM. ASTM C1624-05: Standard test method for adhesion strength and mechanical failure modes of ceramic coatings by quantitative single point scratch testing. Pennsylvania; 2010.

II Holmberg K, Matthews A. Coatings tribology: properties, mechanisms, techniques and applications in surface engineering. 2nd ed. Amsterdam: Elsevier Science; 2009.

Recebido em: 2 Out. 2014

Aceito em: II Fev. 2015 